\title{
Weiterbildung
}

\section{Erste Aktiv-Schulung für Jungzahnmediziner}

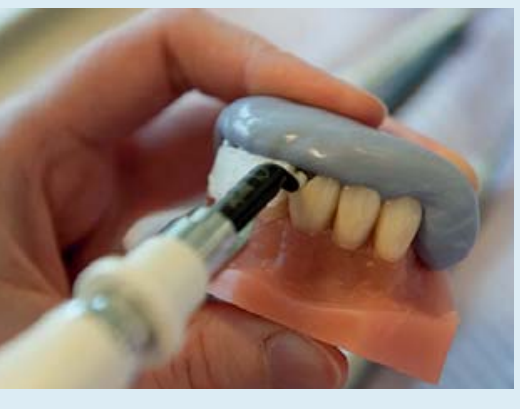

Im Rahmen der 1 . dentXperts assistant Aktiv-Schulung von Heraeus Kulzer entdeckten 20 angehende

Zahnärzte im Oktober die Einsatzmöglichkeiten der Venus-Komposite in der Anwendung des Mix \& Match-Systems. Was sind die Bedürfnisse junger Assistenzzahnärzte? Was hilft ihnen im Praxisalltag? Das Unternehmen hat ein Konzept entwickelt, das Assistenzärzten praktisches Wissen für die unmittelbare Anwendung in der Zahnarztpraxis an die Hand gibt. Christine Dirxen, Zahnärztin aus Berlin und spezialisiert auf ästhetische Zahnheilkunde, teilte ihre Erfahrungen mit den Kursteilnehmern und ließ sich in die Trickschublade schauen. Sie erläuterte die spezielle TCD-Matrix der Nanohybridkomposite des Unternehmens, die breiten Anwen- dungsbereiche von Venus Diamond und des etwas cremigeren Venus Pearl sowie die Applikation in der Einschicht- und Mehrschichttechnik. Zudem wies sie auf die Flexibilität in der Anwendung hin: Die Venus-Komposite sind im Mix \& MatchSystem frei kombinierbar - je nach Indikation und Gewohnheit des Zahnarzts. Wertvolle Tipps erhielten die Jungmediziner auch zu Schichtstärken und Polymerisation. Nach der Pilotveranstaltung in Berlin stehen nun weitere Kurse für Assistenzärzte auf dem Programm. Daneben bietet das Programm vielfältige Angebote für Studenten und Praxisgründer. Als Club-Mitglied können sie entweder zu Sonderkonditionen an Kursen teilnehmen, zahnmedizinische Produkte testen oder sich Unterstützung bei ihrer Praxisgründung sichern. Informieren und kostenlos anmelden können sich junge Zahnmediziner zentral unter www.heraeuskulzer.de/dentxperts.

Nach einer Pressemitteilung der Heraeus Kulzer GmbH, Hanau www.heraeus-kulzer.de 